\title{
TABAQUISMO: UN PROBLEMA QUE AFECTA A JÓVENES UNIVERSITARIOS A NIVEL NACIONAL E INTERNACIONAL
}

TOBACCO: A PROBLEM THAT AFFECTS YOUNG UNIVERSITIES AT THE NATIONAL AND INTERNATIONAL LEVELS

\author{
Lucy E. Correa-López 1,2, Carmen L. Segura-Márquez ,2, Lucy Cedillo-Ramirez 1,2, \\ Andrea M. Morales-Romero ${ }^{1,2}$, Jesus E. Olivera-Ruiz',2
}

\begin{abstract}
Sr. Editor
La Organización Mundial de la Salud define al tabaquismo como una enfermedad adictiva crónica muy extendida a nivel mundial. Representa una de las mayores amenazas para la salud púbica, afectando a niños, jóvenes y adultos'. Se calcula que existen 1.300 millones de fumadores en el mundo y alrededor del $80 \%$ de estos viven en países en los que existe una alta tasa de morbilidad y mortalidad asociada al tabaco principalmente en consumidores adultos ${ }^{2}$.

De manera particular, el consumo de tabaco ente jóvenes universitarios es evidente y preocupante. Diferentes autores en el mundo han planteado factores para el consumo de tabaco entre adolescentes y adultos jóvenes. Tales estudios sostienen que el hábito de fumar inicia, por lo general, durante la adolescencia y se consolida en la etapa universitaria. Además, el tabaquismo en progenitores, familiares y amigos es un factor, quizás el más destacable, que se asocia a la adquisición del hábito tabáquico ${ }^{3}$.

Aunque existen estudios que afirman que el sexo masculino está relacionado con un mayor consumo de tabaco, este ha ido en aumento en la población femenina, especialmente entre las adolescentes y jóvenes adultas que consideran el fumar como un signo de modernidad, libertad e independencia ${ }^{4,5,6}$.

En el Perú, la segunda droga social más consumida es el tabaco. Su consumo comienza frecuentemente en la adolescencia y está determinado por diversos factores socioculturales y personales, que en muchos casos deviene en adicciones y en la adquisición de enfermedades que en su mayoría tienen consecuencias mortales7.

Las estadísticas descritas evidencian que el hábito tabáquico existe entre los adolescentes y adultos jóvenes. Aunque no es determinante, el contexto universitario podría convertirse en un refuerzo social donde se promuevan comportamientos que induzcan a su consumo ${ }^{8}$. Por ello, nuestro equipo de investigación se encuentra estudiando los factores de riesgo asociados al consumo de tabaco en jóvenes universitarios, los resultados preliminares evidencian la necesidad de profundizar en su estudio y desarrollar programas que promuevan estilos de vida saludables, recordando que los universitarios de hoy serán los profesionales del futuro.
\end{abstract}

Contribuciones de autoría: Los autores participaron en la Conflicto de interés: Los autores declaran no tener generación, recolección de información, redacción y versión conflictos de interés en la publicación de este artículo. final del artículo original.

Recibido: 16 de marzo 2019

Financiamiento: Autofinanciado.

Aprobado: 16 de mayo 2019

Correspondencia: Lucy E. Correa-López/Dirección:Av. Alfredo Benavides 5440, Santiago de Surco 15039, Lima-Perú / Teléfono: 970922865 / E-mail: lucyecl@hotmail.com

\section{REFERENCIAS BIBLIOGRÁFICAS}

1.Tabaco [Internet]. [citado 4 de abril de 2019]. Disponible en: https:// www.who.int/es/news-room/fact-sheets/detail/tobacco

2.Atlas Mundial de la Salud [Internet]. [citado 4 de abril de 2019]. Disponible en: http://www.atlasdelasalud.org/

3.Muñoz-Pindado C, Muñoz-Pindado C, Roura-Poch P, Riesco-Miranda JA, Muñoz-Méndez J. Prevalencia del tabaquismo en los estudiantes de bachillerato de la Comarca de Osona (Barcelona). Med Fam SEMERGEN [Internet]. diciembre de 2018 [citado 4 de abril de 2019]; Disponible en: https://linkinghub.elsevier.com/retrieve/pii/S1138359318304337

4.Cheesman Mazariegos SS, Suárez Lugo N. Factores de riesgo y protectores del tabaquismo en estudiantes de la Facultad Ciencias Médicas USAC, Guatemala. Horiz Sanit. 2014;13(3):223-32.
5.Ríos LAR-, Camacho-Rodríguez DE, Ballestas LFF-, Ferrel-Ortega FR, Bautista-Pérez F. Diferencias en el consumo de tabaco en estudiantes según el sexo universitarios. Rev Cuba Enferm [Internet]. 28 de diciembre de 2018 [citado 4 de abril de 2019];34(3). Disponible en: http://www. revenfermeria.sld.cu/index.php/enf/article/view/1479

6.Jiménez Rodrigo ML. Mujeres y tabaco la feminización del consumo de cigarrillos en España. [Granada]: Editorial de la Universidad de Granada; 2007. 7.CEDRO. El Problema de las Drogas en el Perú 2018 [Internet]. 2018 [citado 5 de abril de 2019]. Disponible en: http://181.65.165.78/handle/ CEDRO/378

8.Novoa-Gómez MM, Barreto I, Manuel Silva L. Consumo de cigarrillo y prácticas culturales en contextos universitarios. Rev Latinoam Psicol. abril de 2012;44(1):97-110.

${ }^{1}$ Facultad de Medicina Humana, Universidad Ricardo Palma, Lima-Perú.

${ }^{2}$ Instituto de Investigación en Ciencias Biomédicas, Universidad Ricardo Palma, Lima-Perú.

citar como: Lucy E. Correa-López, Carmen L. Segura-Márquez, Lucy Cedillo-Ramirez, Andrea M. Morales-Romero, Jesus E. Olivera-Ruiz. Tabaquismo: un problema que afecta a jóvenes universitarios a nivel nacional e internacional. Rev. Fac. Med. Hum. Julio 2019; 19(3):106. DOI 10.25176/RFMH.v19i3.2147 